\title{
Uncertainty Models and Robust Complex-Rational Controller Design for Flexible Structures
}

\author{
Nabil Aouf* \\ National Research Council of Canada, Boucherville, Québec J4B 6Y4, Canada \\ and \\ Benoit Boulet ${ }^{\dagger}$ \\ McGill University, Montréal, Québec H3A 2A7, Canada
}

\begin{abstract}
Real parametric uncertainties in the modal damping ratios and frequencies of flexible structures are represented by complex uncertainties that can lead to robust controller designs satisfying robust performance specifications. These complex uncertainty blocks are useful in a $\mu$-synthesis controller design procedure. Two models are proposed for modal parameter uncertainties. The first model uses a coprime factorization representation of the perturbed plant, whereas in the second model, a diagonal representation with complex eigenvalues is used. The innovation in the second method proposed is the use of a complex-rational controller design strategy, which offers tight uncertainty bounds and leads to a robust performance controller. The frequency response of the complex-rational controller is then approximated by a real-rational controller achieving the robust performance specifications.
\end{abstract}

\section{Introduction}

$\mathbf{F}$ LEXIBLE structures are generally characterized by the undamped natural frequencies and damping ratios of their flexible modes. These parameters are subject to errors when they are estimated. Such uncertainties are important and should be taken into account in a robust controller design. The proper capture of modal parameter uncertainties in dynamic models of flexible structures for robust control has been the subject of ongoing efforts. Previous research ${ }^{1,2}$ used additive or multiplicative uncertainty models to take into account the variation in the dynamics of the plant. Another way is to use certain heuristics to facilitate the representation of the parametric uncertainties in the flexible modes by a parametric model. ${ }^{3}$ These heuristics represent approximations in the parameter variation that are not generally realistic and lead to conservative controller designs, that is, designs that cannot provide the desired performance in the face of realistic levels of parametric uncertainty.

Recently, a model representing parametric uncertainties in the modes of a flexible structure was discussed in Ref. 4. Note that such models were developed ${ }^{5}$ a few years ago. In Ref. 5, a model of dynamic uncertainty covering parametric variations in the flexible modes of a flexible structure was developed. This dynamic uncertainty has the virtue of being nonconservative, but only when the frequencies of the flexible modes are close to each other. In this paper, we propose to represent perturbations in the frequency and damping ratio of each flexible mode by a tight low-order dynamic uncertainty. Thus, we reduce the order of the augmented plant by half and transform the mixed real/complex uncertainty robust performance $\mu$-synthesis problem into an easier complex $\mu$ synthesis. We use two techniques: The first is based on the coprime factorization framework, ${ }^{6}$ and the second uses a complex diagonal modal representation to model the dynamics of the flexible structure and

Received 27 July 2001; revision received 14 October 2002; accepted for publication 15 February 2003. Copyright (C) 2003 by Nabil Aouf and Benoit Boulet. Published by the American Institute of Aeronautics and Astronautics, Inc., with permission. Copies of this paper may be made for personal or internal use, on condition that the copier pay the $\$ 10.00$ per-copy fee to the Copyright Clearance Center, Inc., 222 Rosewood Drive, Danvers, MA 01923; include the code 0731-5090/03 \$10.00 in correspondence with the CCC.

* Researcher, Intelligent Forming Technologies Group, Industrial Materials Institute, 75 de Mortagne Boulevard; nabil.aouf@nrc.ca.

${ }^{\dagger}$ Assistant Professor, Department of Electrical and Computer Engineering and Director of the Industrial Automation Laboratory, Centre for Intelligent Machines; boulet@cim.mcgill.ca. Member AIAA. to take into account the parametric uncertainties. One advantage of our methods consists in transforming the real modal parametric uncertainties into a smaller number of complex uncertainty blocks. These cover all damping and frequency variations in the flexible structure and reduce the complexity of the augmented plant.

The robust controller design we propose takes into account two aspects: robustness to the uncertainties in the modal parameters and the closed-loop performance specified for our model. The second uncertainty model proposed results in a complex-rational nominal plant. This led us to develop a new procedure to design a robust controller. A robust complex-rational controller is first obtained via a $\mu$ synthesis, and then a real-rational controller keeping the robust performance level is designed to approximate the frequency response of the complex-rational controller.

\section{Problem Setup}

Because of the lack of exact knowledge of the undamped natural frequencies and damping ratios, the control designer needs to estimate uncertainty bounds for the modal parameters $\zeta_{i}$ and $\omega_{i}$, $i=1, \ldots, n$. These bounds are used in the design to achieve the required robustness. Let the perturbed modal parameters be written as $\zeta_{i p}=\zeta_{i}+\delta_{\zeta_{i}}$ and $\omega_{i p}=\omega_{i}+\delta_{\omega_{i}}$, where $\zeta_{i}$ and $\omega_{i}$ are the $i$ th nominal damping ratio and frequency, respectively. The real numbers $\delta_{\zeta_{i}}$ and $\delta_{\omega_{i}}$ represent the uncertainty in each parameter and are bounded in magnitude by $\left|\delta_{\omega_{i}}\right|<t_{\omega_{i}}$ and $\left|\delta_{\zeta_{i}}\right|<t_{\zeta_{i}}$. These uncertainties have to be taken into account in the design of a robust controller. One of the most used and efficient design approaches to achieve robust performance for a mixed type of uncertainty, that is, a combination of real and complex perturbations, is the mixed $\mu$ design based on the $D, G-K$ iteration algorithm (a successive minimization of $\mu$ over $D, G$ scales and controllers). ${ }^{?}$

In our control design method, we consider the parametric perturbations $\delta_{\zeta_{i}}$ and $\delta_{\omega_{i}}$ for each flexible mode. For these perturbations, no tight, realistic, and efficient (for control design) parametric model is available in the literature. Consequently, it is difficult to deal with real uncertainty representations to achieve closed-loop robust performance criteria. Moreover, an increase in the number of scalar perturbations causes a problem of dimensional complexity in the controller design's $D, G-K$ algorithm. The order of the plant augmented with the $D, G$ scales can be very high, leading to potential numerical problems in the design. This difficulty is compounded with the order of the nominal model of the flexible structure, which is typically high. Even though the $\mu$-synthesis procedure used here (based on successive $\mathcal{H}_{2}$ designs, cf. Ref. 8) reduces the dimension of the scales by half, a completely satisfactory solution to the tight 
covering of parametric uncertainty has yet to be found. A novel way to deal with this problem, under a new concept of complex controller design, is proposed here.

\section{Coprime Factorization Approach}

This is the uncertainty modeling technique inspired and adapted from Ref. 4. Consider the nominal dynamic equation of the flexible structure, with $m$ inputs and $p$ outputs, in modal coordinates:

$$
\ddot{\eta}+D \dot{\eta}+\Lambda \eta=B u, \quad y=C \eta
$$

where $\quad D:=\operatorname{diag}\left\{2 \zeta_{i} \omega_{i}, \ldots, 2 \zeta_{n} \omega_{n}\right\} \in \mathbb{R}^{n \times n}, \quad \Lambda:=\operatorname{diag}\left\{\omega_{1}^{2}, \ldots\right.$, $\left.\omega_{n}^{2}\right\} \in \mathbb{R}^{n \times n}, B \in \mathbb{R}^{n \times m}$, and $C \in \mathbb{R}^{p \times n}$. Taking the Laplace transform of Eq. (1), we obtain

$$
\eta(s)=\left(s^{2} I+s D+\Lambda\right)^{-1} B u(s), \quad y(s)=C \eta(s)
$$

Let us define $G(s):=\left(s^{2} I+s D+\Lambda\right)^{-1}$ and let the polynomial $s^{2}+a s+b$ be Hurwitz with real zeros that are typically selected at slightly higher frequency than the flexible modes. Transfer matrix $G$ can be written as $G=\tilde{M}^{-1} \tilde{N}$, where

$$
\begin{aligned}
\tilde{M}:= & {\left[1 /\left(s^{2}+a s+b\right)\right] } \\
& \times \operatorname{diag}\left\{s^{2}+2 \zeta_{1} \omega_{1} s+\omega_{1}^{2}, \ldots, s^{2}+2 \zeta_{n} \omega_{n} s+\omega_{n}^{2}\right\} \\
\tilde{N}:= & \operatorname{diag}\left\{\left[1 /\left(s^{2}+a s+b\right)\right], \ldots,\left[1 /\left(s^{2}+a s+b\right)\right]\right\}
\end{aligned}
$$

Note that $\tilde{M}$ and $\tilde{N}$ form a left-coprime factorization of $G$ in $\mathbb{R} \mathcal{H}_{\infty}$, the space of stable real-rational transfer matrices. The perturbed plant can be written as

$$
G_{p}:=\left(\tilde{M}+\Delta_{\tilde{M}}^{r}\right)^{-1}\left(\tilde{N}+\Delta_{\tilde{N}}^{r}\right)
$$

where $\Delta_{\tilde{M}}^{r}$ and $\Delta_{\tilde{N}}^{r} \in \mathbb{R} \mathcal{H}_{\infty}$ cover all real parametric perturbations in the flexible modes and

$$
\begin{aligned}
\Delta_{\tilde{M}}^{r} & :=\operatorname{diag}\left\{\frac{\left[2 \zeta_{1} \delta_{\omega_{1}}+2 \delta_{\zeta_{1}}\left(\omega_{1}+\delta_{\omega_{1}}\right)\right] s+2 \omega_{1} \delta_{\omega_{1}}+\delta_{\omega_{1}}^{2}}{s^{2}+a s+b},\right. \\
& \left.\ldots, \frac{\left[2 \zeta_{n} \delta_{\omega_{n}}+2 \delta_{\zeta_{n}}\left(\omega_{n}+\delta_{\omega_{n}}\right)\right] s+2 \omega_{n} \delta_{\omega_{n}}+\delta_{\omega_{n}}^{2}}{s^{2}+a s+b}\right\} \\
\Delta_{\tilde{N}}^{r} & :=\operatorname{diag}\{0, \ldots, 0\}
\end{aligned}
$$

Note that the perturbation on factor $\tilde{N}$ could be used to represent mode shape uncertainty, although this is not pursued here.

$$
\begin{aligned}
G_{p} & :=\left(\tilde{M}+\Delta_{\tilde{M}}^{c}\right)^{-1} \tilde{N} \\
& =\text { blockdiagonal }\left\{\left[\begin{array}{c}
s+\omega_{i}\left(\zeta_{i}-j\right)+\omega_{i} \delta_{\zeta_{i}}+\delta_{\omega_{i}}\left(\zeta_{i}-j\right)+\delta_{\omega_{i}} \delta_{\zeta_{i}} \\
0
\end{array}\right.\right. \\
\Delta_{\tilde{M}}^{c} & :=\frac{1}{s+\alpha} \text { blockdiagonal }\left\{\Delta_{\tilde{M}_{i}}^{c}\right\}_{i=1}^{n} \\
\Delta_{\tilde{M}_{i}}^{c} & :=\left[\begin{array}{cc}
\omega_{i} \delta_{\zeta i}+\delta_{\omega_{i}}\left(\zeta_{i}-j\right)+\delta_{\omega_{i}} \delta_{\zeta i} & \omega_{i} \delta_{\zeta_{i}}+\delta_{\omega_{i}}\left(\zeta_{i}+j\right)+\delta_{\omega_{i}} \delta_{\zeta_{i}}
\end{array}\right] \\
0 &
\end{aligned}
$$

bounded by the magnitudes of the diagonal entries of the following weighting function (see example 1):

$$
\begin{gathered}
W_{\tilde{M}}:=\operatorname{diag}\left\{\frac{\left[2 \zeta_{1} t_{\omega_{1}}+2 t_{\zeta_{1}}\left(\omega_{1}+t_{\omega_{1}}\right)\right] s+2 \omega_{1} t_{\omega_{1}}+t_{\omega_{1}}^{2}}{s^{2}+a s+b},\right. \\
\left.\ldots, \frac{\left[2 \zeta_{n} t_{\omega_{n}}+2 t_{\zeta_{n}}\left(\omega_{n}+t_{\omega_{n}}\right)\right] s+2 \omega_{n} t_{\omega_{n}}+t_{\omega_{n}}^{2}}{s^{2}+a s+b}\right\}
\end{gathered}
$$

The resulting bounds represent structured complex blocks of uncertainty. These uncertainty bounds are more general than the single bound obtained in Ref. 5, which was ultimately for unstructured uncertainty and which used the assumption that the modal frequencies of the flexible structure were close to each other.

\section{Modal Coordinates Approach}

Suppose that the state-space model in modal coordinates of a flexible structure (or a flexible aircraft) is given as

$$
\dot{x}=A x+B u, \quad y=C x+D u
$$

where $A=$ blockdiagonal $\left\{A_{i}\right\}_{i=1}^{n}$,

$$
A_{i}=\left[\begin{array}{cc}
-\zeta_{i} \omega_{i}+j \omega_{i} & 0 \\
0 & -\zeta_{i} \omega_{i}-j \omega_{i}
\end{array}\right]
$$

Note that the $A$ matrix is complex, that is, $A \in \mathbb{C}^{2 n \times 2 n}$. We use the approximation $\zeta_{i} \ll 1$, which is verified in many examples of flexible structure models. Let us define the complex-rational factorization in $\mathbb{C H}_{\infty}$ :

$$
G:=(s I-A)^{-1}=\tilde{M}^{-1} \tilde{N}
$$

where $\tilde{M}:=\operatorname{diag}\left\{\tilde{M}_{i}\right\}_{i=1}^{n} \in \mathbb{C} \mathcal{H}_{\infty}$,

$$
\begin{aligned}
\tilde{M}_{i} & :=\frac{1}{s+\alpha}\left(s I-A_{i}\right) \\
& =\frac{1}{s+\alpha}\left[\begin{array}{cc}
s+\omega_{i} \zeta_{i}-j \omega_{i} & 0 \\
0 & s+\omega_{i} \zeta_{i}+j \omega_{i}
\end{array}\right] \\
\tilde{N} & :=\operatorname{diag}\left\{\frac{1}{s+\alpha}, \ldots, \frac{1}{s+\alpha}\right\} \in \mathbb{C} \mathcal{H}_{\infty}
\end{aligned}
$$

and $s+\alpha$ is Hurwitz with its zero typically chosen at a slightly higher frequency than the flexible modes. The perturbed model can be written as
Each nonzeroentry of $\Delta_{\tilde{M}}^{r}$, representing a family of strictly proper transfer functions, can be tightly bounded with a second-order weighting function using the upper bounds $t_{\zeta_{i}}$ and $t_{\omega_{i}}, i=1, \ldots, n$, on the parameter uncertainties, that is, the diagonal entries of $\Delta_{\tilde{M}}^{r}$ are
The magnitude of each subblock $\Delta_{\tilde{M}_{i}}^{c}$ can be tightly bounded by using the maximum value of each modal parameter, $t_{\zeta_{i}}$ and $t_{\omega_{i}}$. Because we are interested in the magnitude of the uncertainty that the system is subjected to, it is possible to use the complex-rational 
weighting function $W_{\tilde{M}}$ to bound $\Delta_{\tilde{M}}$ in the design (see example 2):

$W_{\tilde{M}}:=\frac{1}{s+\alpha}$ blockdiagonal

$\times\left\{\left[\begin{array}{cc}\omega_{i} t_{\zeta_{i}}+t_{\omega_{i}} \zeta_{i}+t_{\omega_{i}} t_{\zeta_{i}}-j t_{\omega_{i}} & 0 \\ 0 & \omega_{i} t_{\zeta_{i}}+t_{\omega_{i}} \zeta_{i}+t_{\omega_{i}} t_{\zeta_{i}}+j t_{\omega_{i}}\end{array}\right]\right\}_{i=1}^{n}$

The flexible mode uncertainty blocks in the design will be tightly covered in magnitude by entries on the main diagonal of $W_{\tilde{M}}$.

\section{Control Design}

Our control design methodology is based on the coprime factorization obtained from either uncertainty representation introduced earlier. We use the $\mu$-design technique to take into account the robust performance specification. The design concept is explained by Fig. 1. Robust performance is taken into account in the $\mu$ design by including a fictitious uncertainty $\Delta_{p}$ linking the input $r$ to the outputs $z_{1}$ and $z_{2}$. We transform the scheme given in Fig. 1 to the classical $\mu$ setup. We obtain the design given in Fig. 2, where

$$
\begin{gathered}
z:=\left[\begin{array}{lll}
v & z_{1}^{T} & z_{2}^{T}
\end{array}\right]^{T}, \quad w:=\left[\begin{array}{ll}
w_{\Delta}^{T} & r^{T}
\end{array}\right]^{T}, \quad \Delta:=\left[\begin{array}{cc}
\bar{\Delta}_{\tilde{M}} & 0 \\
0 & \Delta_{p}
\end{array}\right] \\
P=\left[\begin{array}{ccc}
\tilde{M}^{-1} W_{\tilde{M}} & 0 & \tilde{N} \\
-W_{p} C \tilde{M}^{-1} W_{\tilde{M}} & -W_{p} & -W_{p} \tilde{N} B \\
0 & 0 & W_{u} \\
C \tilde{M}^{-1} W_{\tilde{M}} & 0 & \tilde{N} B
\end{array}\right]
\end{gathered}
$$

Note that $\bar{\Delta}_{\tilde{M}}:=$ blockdiagonal $\left\{\bar{\Delta}_{\tilde{M}_{i}}\right\}_{i=1}^{n}$ is normalized to be less than one in the norm, such that the uncertainty set can be defined as

$$
\begin{aligned}
\mathcal{D}:= & \left\{\Delta_{\tilde{M}} \in \mathcal{H}_{\infty}: \Delta_{\tilde{M}}\right. \\
& \left.=W_{\tilde{M}} \bar{\Delta}_{\tilde{M}}, \bar{\Delta}_{\tilde{M}_{i}} \in \mathcal{H}_{\infty},\left\|\bar{\Delta}_{\tilde{M}_{i}}\right\|_{\infty}<1, i=1, \ldots, n\right\}
\end{aligned}
$$

This is a set of structured stable perturbations (blockdiagonal) that contains the real-rational or complex-rational perturbations introduced earlier that cover the parameter perturbationsin each flexible mode.

One advantage that this uncertainty model offers is that we can cover the structured complex uncertainties using a single full block.

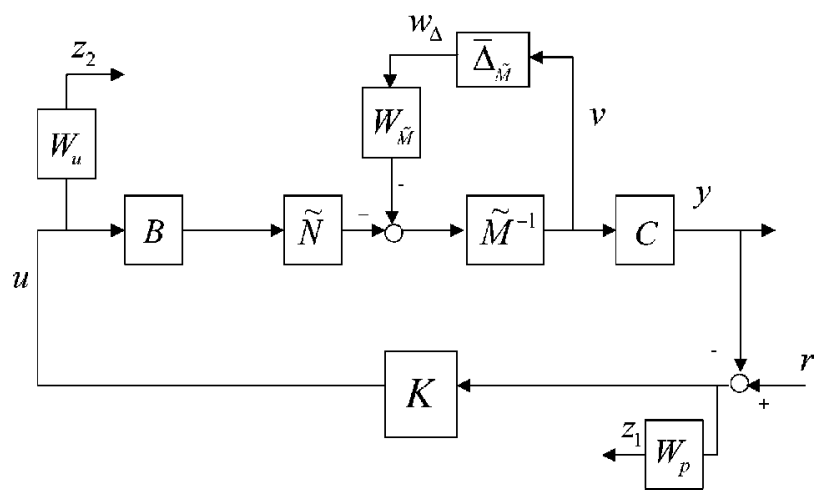

Fig. 1 Setup for design of robust controller.

Fig. 2 Setup for $\mu$ synthesis.

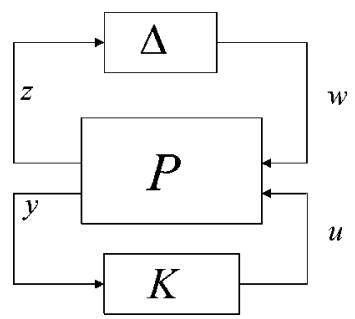

In fact, for the frequency interval considered, the highest magnitude uncertainty bound can be chosen to cover in the norm the set of unstructured uncertainties, which contains the blockdiagonal perturbations derived earlier. This approach, although conservative, simplifies the design of a $\mu$ controller.

\section{Simulation and New Control Design Strategy}

To validate our methods, we chose two flexible systems based on the well-known three-mass example. ${ }^{9}$

\section{Example 1}

In the first example, the damping ratios and the frequencies of the flexible modes are $\zeta_{1}=0.072, \zeta_{2}=0.023, \zeta_{3}=0.016$, $\omega_{1}=0.91, \omega_{2}=1.81$, and $\omega_{3}=1.5$. The input matrix $B$ is given by $B=\left[\begin{array}{lll}0 & 2.4 & 4.4\end{array}\right]^{T}$. We assume that the uncertainties in $\zeta_{i}$ and $\omega_{i}, i=1,2,3$, are 10 and $0.1 \%$, respectively. Thus, the controller to be designed has to be more robust against variations in the damping ratios. The level of performance specified is given by $W_{p}=(s+3) /(10.5 s+0.03)$, and the constraint on the controlleris specified by $W_{u}=1 / 0.7$. In this example, we use the first approach described. Figure 3 are Bode plots of the first diagonal entry in $\Delta_{\tilde{M}}^{r}$ for modal parameter variations over their intervals in the first flexible mode and the magnitude of the first diagonal entry of $W_{\tilde{M}}$. It is clear that this magnitude tightly bounds all of the variations in the first diagonal entry of $\Delta_{\tilde{M}}^{r}$. This observation is valid for the other flexible modes as well. Thus, the weighting function $W_{\tilde{M}}$ constitutes a tight upper bound on $\Delta_{\tilde{M}}^{r}$.

A $\mu$ controller was designed for this example taking into account the complex uncertainty $W_{\tilde{M}}$ covering all possible parameter variations in all of the flexible modes. Figure 4 shows the upper bound on $\mu$ that we could get with this controller. The $\mu$ upper bound obtained is less than 0.72 , which shows that this controller design effectively

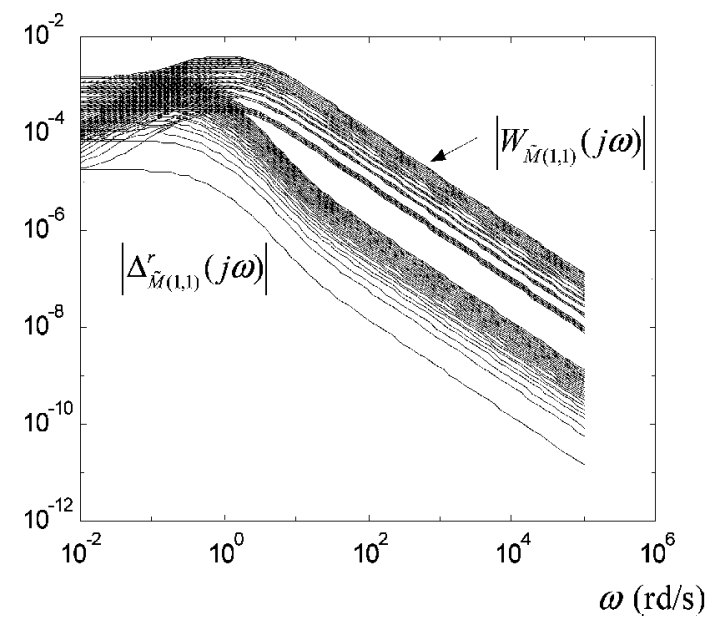

Fig. 3 Upper bound on magnitudes of perturbations for example 1.

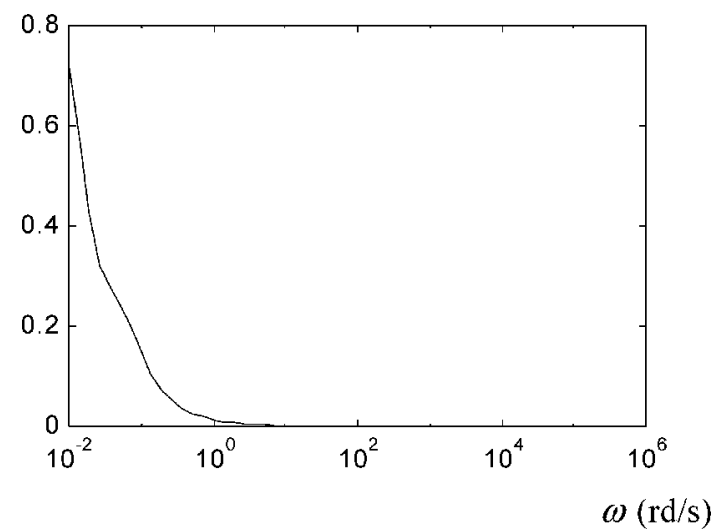

Fig. 4 Upper bound on $\mu$ for example 1. 


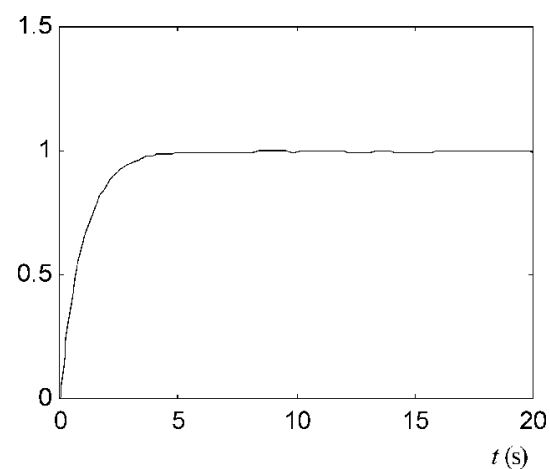

Fig. 5 Step response for example 1.

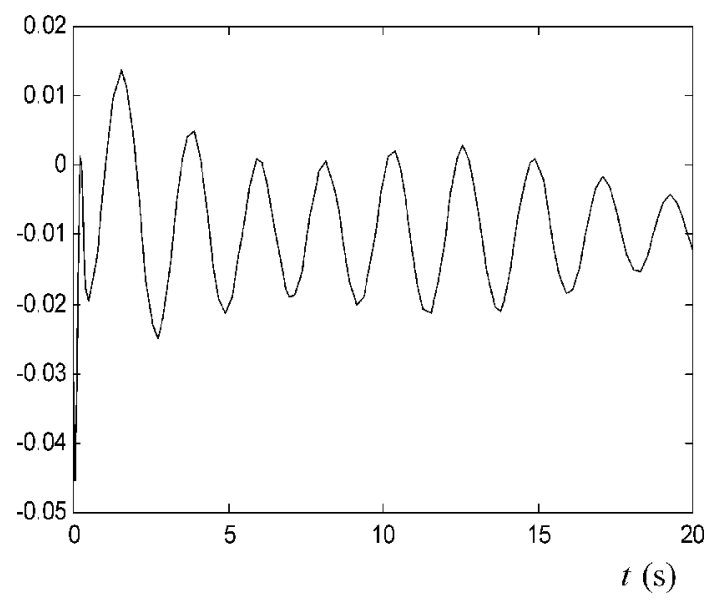

Fig. 6 Controller output for example 1.

guarantees the robust performance specified, in spite of parameter uncertainties in the flexible modes. Note that obtaining the performance index $\mu$ less than one means that the designed $\mu$ controller gives the performance desired over all possible parameter variations in damping ratios and modal frequencies.

A step response of the perturbed system ( $+5 \%$ for damping ratios and $+0.1 \%$ natural frequencies) was simulated. Figures 5 and 6 show a good tracking response and a reasonable controller action over time, respectively, which confirm the results in the frequency domain given by the $\mu$ upper bound.

\section{Example 2}

The second example representing the three-mass single-input/ single-output system is given in a complex modal representation as described earlier. The damping ratios and frequencies of the flexible modes are $\zeta_{1}=0.025, \zeta_{2}=0.012, \zeta_{3}=0.0043, \omega_{1}=5.12$, $\omega_{2}=2.43$, and $\omega_{3}=0.87$. The uncertainties that the damping ratios and frequencies are subjected to are 10 and $3 \%$, respectively. These uncertainty levels, especially in the modal frequencies, are quite demanding for a robust control design. The weighting functions $W_{p}$ and $W_{u}$ are the same as the ones used in the first example. Figure 7 shows that the magnitude of the first element in $W_{\tilde{M}}$ tightly bounds all variations in the first entry on the diagonal of $\Delta_{\tilde{M}}^{c}$.

The $\mu$-synthesis approach used for the complex-rational controller design is based on the method of successive scaled $\mathcal{H}_{2}$ optimization problems. ${ }^{8}$ These optimizations are treated and solved by means of two Riccati equations over the field of complex numbers:

$$
\begin{aligned}
(A- & \left.B_{2} R_{1}^{-1} D_{12}^{*} C_{1}\right)^{*} X+X\left(A-B_{2} R_{1}^{-1} D_{12}^{*} C_{1}\right) \\
& -X B_{2} R_{1}^{-1} B_{2}^{*} X+C_{1}^{*}\left(I-D_{12} R_{1}^{-1} D_{12}^{*}\right) C_{1}=0 \\
(A- & \left.B_{1} D_{21}^{*} R_{2}^{-1} C_{2}\right)^{*} Y+Y\left(A-B_{1} D_{21}^{*} R_{2}^{-1} C_{2}\right) \\
& -Y C_{2} R_{2}^{-1} C_{2}^{*} Y+B_{1}^{*}\left(I-D_{21}^{*} R_{2}^{-1} D_{21}\right) B_{1}^{*}=0
\end{aligned}
$$

where $R_{1}=D_{12}^{*} D_{12}$ and $R_{2}=D_{21} D_{21}^{*}$.

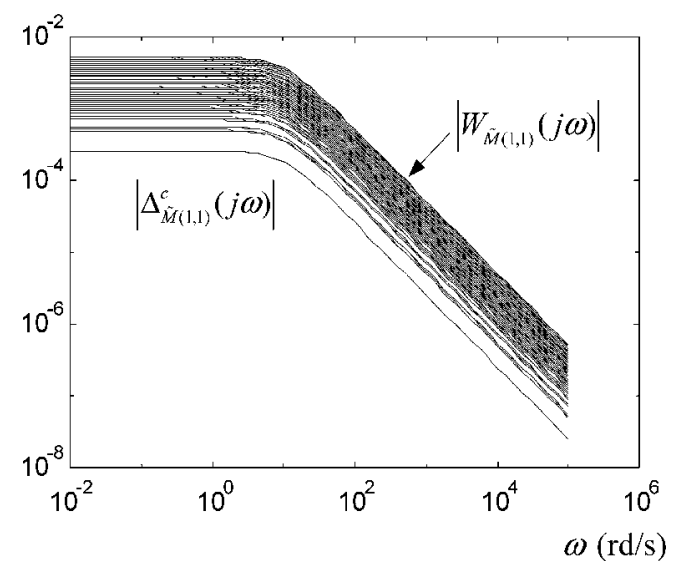

Fig. 7 Upper bound on magnitudes of perturbations for example 2.

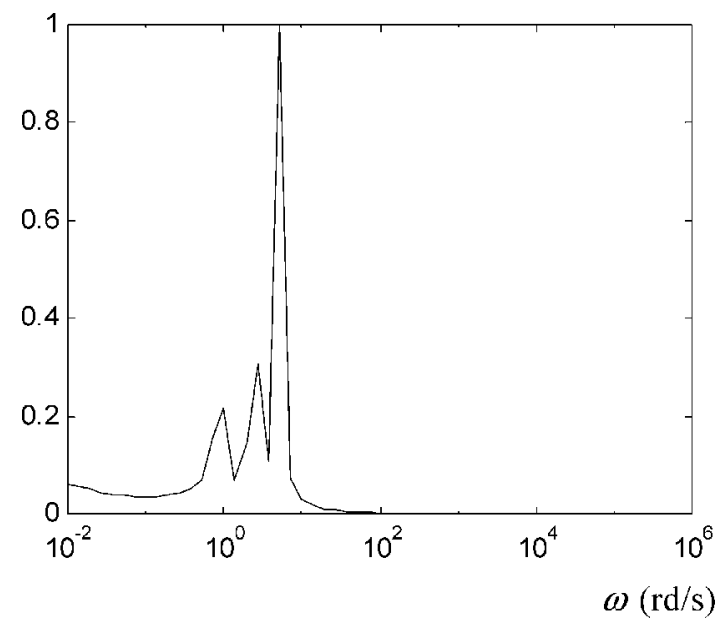

Fig. 8 Upper bound on $\mu$ with complex-rational controller for example 2.

The scaled augmented plant $P$ in its simplified state-space representation is given by

$$
P:=\left[\begin{array}{c|cc}
A & B_{1} & B_{2} \\
\hline C_{1} & 0 & D_{12} \\
C_{2} & D_{21} & 0
\end{array}\right]
$$

where $A \in \mathbb{C}^{n \times n}, \quad B_{1} \in \mathbb{C}^{n \times n_{w}}, \quad B_{2} \in \mathbb{C}^{n \times n_{u}}, C_{1} \in \mathbb{C}^{n_{z} \times n}, \quad C_{2} \in$ $\mathbb{C}^{n_{y} \times n}, D_{12} \in \mathbb{C}^{n_{z} \times n_{u}}$, and $D_{21} \in \mathbb{C}^{n_{y} \times n_{w}}$. Because the state matrices involved in the Riccati equations are complex, there is the possibility of designing a complex-rational $\mu$ controller achieving the performance and robustness specifications required.

The complex-rational $\mu$ controller designed in this case achieved the specified robust performance criterion. Figure 8 shows the upper bound on $\mu$ obtained for this design. Its maximum equals 0.99 . Note that the $\mu$ controlleris not optimal; this is because of the uncertainty block structure. This problem of suboptimality of a $\mu$ design has been mentioned in the literature. ${ }^{7,8}$ However, the $\mu$ design still appears to be the best strategy to deal with structured uncertainties in practice. The complex-rational controller design allowed us to deal efficiently with the parametric uncertainties under a complex representation. An uncertainty of up to $3 \%$ in the modal frequencies could be tolerated, a level rarely reported in the literature.

After designing a complex-rational controller achieving the robust performance specification, we have to recover a realizable realrational controller. This controller has to maintain the performance level obtained by the complex-rational controller. The problem can be posed as

$$
\min _{K_{\mathrm{Re}}}\left\|K-K_{\mathrm{Re}}\right\|_{\infty}
$$


where $K$ is the complex-rationalcontroller designed and $K_{\mathrm{Re}}$ is the real-rational controller to be found. By enforcing closeness of the frequency responses of the two controllers using the infinity norm, we can ensure that both controllers will act in the same manner on the generalized plant when closing the feedback loop. Thus, the level of performance obtained by the complex-rational controller can be approached by using a real-rational controller. One way to solve the preceding minimization problem is to fit the magnitude and phase of the complex-rational controller's frequency response by a real-rational system $K_{\mathrm{Re}}$. Tools that have the ability to provide a solution in this fitting procedure may be found in the MATLAB ${ }^{\circledR}$ Identification toolbox..$^{10}$ One of the benefits of this design procedure is the reduction of the real-rationalcontroller.In fact, the typicalconstraint of maintaining the structure of the original controllerin most classical reduction procedures is not present in the fitting procedure proposed here: The structure of the original complex-rational controller and the reduced real-rational controller may differ. Thus, a low-order, real-rationalcontroller achieving the robust performance specification can be obtained.

In fact, enforcing the closeness of the complex-rationalcontroller and the real controller in the frequency domain is just a heuristic proposed here to obtain a real controller. Future work will automate the procedure of obtaining a real-rational controller without using a trial-and-errormethod. A real-rational controller of order 2, achieving a robust performance level of 0.85 , was generated for our example. The fit of a low-order, real-rational controller frequency response on the complex-rationalcontroller's frequency response is presented in Fig. 9.

The robust performance level for the real controller is shown in Fig. 10. The robust complex-rational controller is of order 28. This high order resulted from the number and order of the scales in the $\mu$ design. Because of the nonoptimality of the $\mu$ design, the robust

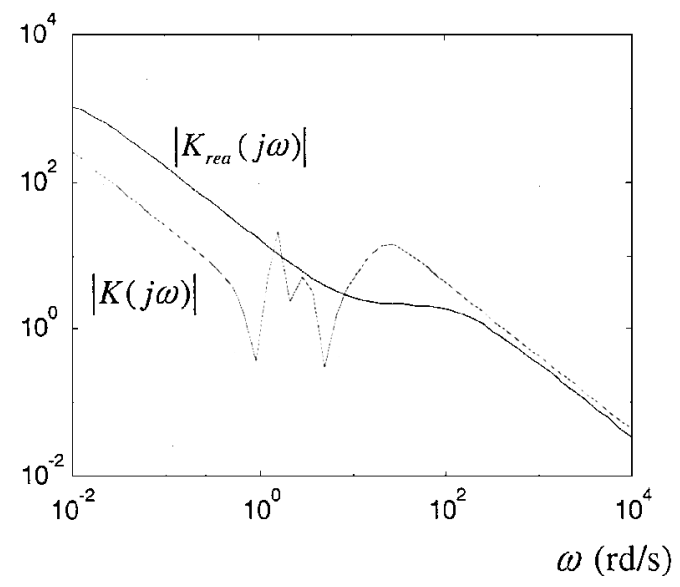

Fig. 9 Fitting procedure results.

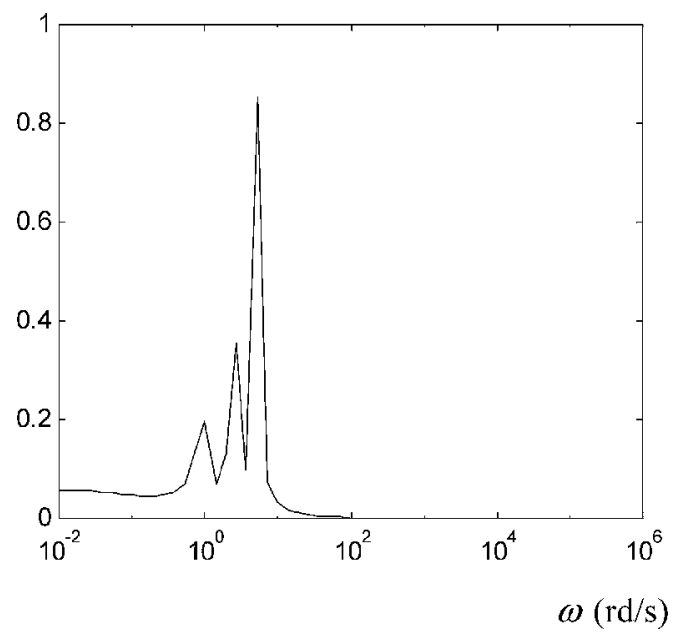

Fig. 10 Upper bound on $\mu$ with real-rational controller for example 2. performance of the second-order,real-rational controller was better than the one obtained by the original complex-rational controller.

Time simulation of the perturbed closed-loop system ( $+5 \%$ for all damping ratios and $+3 \%$ for all natural frequencies) with the designed real-rational controller is presented in Figs. 11 and 12. It is shown that the tracking response is acceptable with a reasonable control effort.

To compare both proposed methods, we applied the first method to example 2. We constrained the controller design with 1 and $0.3 \%$

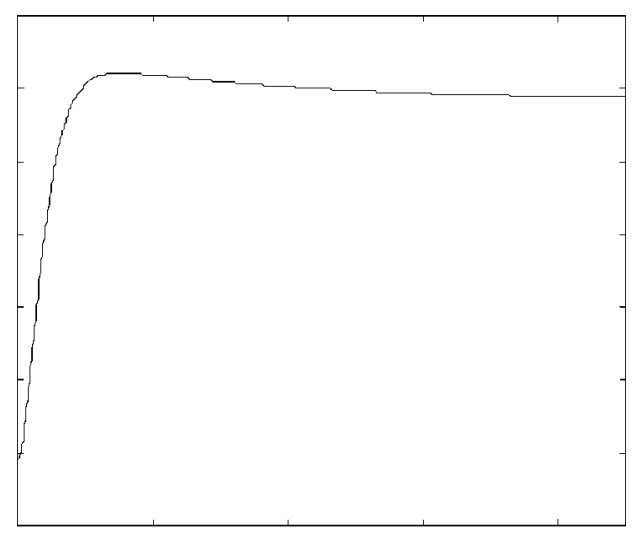

$t(\mathrm{~s})$

Fig. 11 Step response for example 2 with real-rational controller.

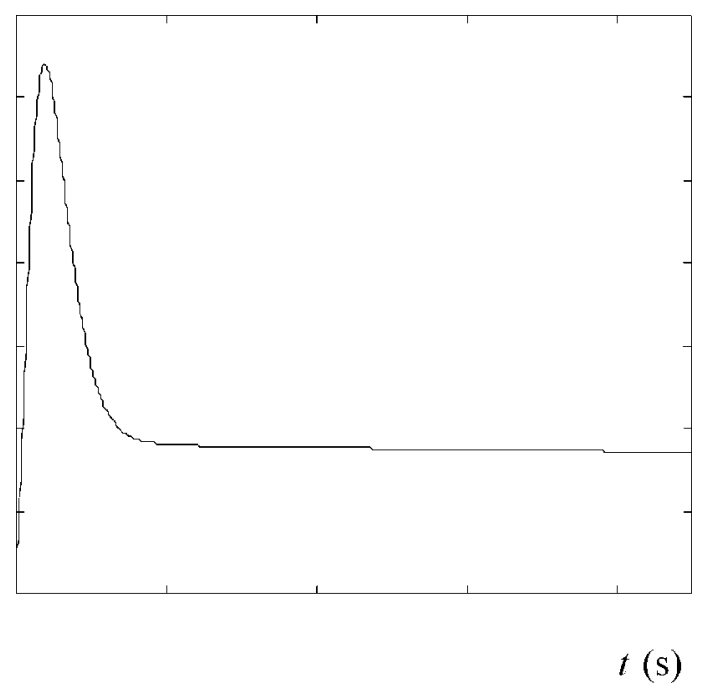

Fig. 12 Controller output for example 2 with real-rational controller.

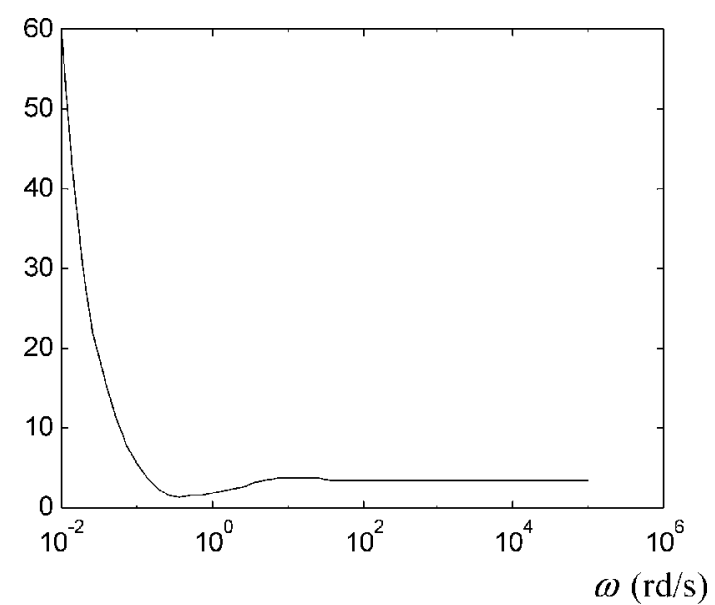

Fig. 13 Example 2: $\mu$ upper bound of the coprime factor approach. 
uncertainties on the damping ratios and the frequency response. Even though the level of uncertainty is less than that of the modal coordinate approach, a robust controller could not be found. The $\mu$ upper bound obtained with the designed controller is presented in Fig. 13. It shows that $\mu$ upper bound is much greater than one. Thus, robust performance is not achieved with the coprime factorization approach. We cannot generalize that modal coordinate approach is better than the coprime factorization approach. However, we can confirm that the modal coordinate approach is suitable for a robust controller design and gives better results than the coprime factorization approach, at least for this example.

\section{Conclusions}

New control-orientedmodels to represent parametric uncertainty in the flexible modes of flexible structures are proposed. Perturbations of real-rational and complex-rational coprime factor models are used in a $\mu$-synthesis design that reduce the order of the augmented plant. This in turn reduces the order of the robust controller. The modal complex-rational approach leads to a complex-rational controller design that is not directly implementable. However, a method to obtain a real-rational controller keeping the robust performance level of the complex-rational controller was discussed. The real-rational controller is designed such that its frequency response is close to that of the complex controller, with the possibility of keeping its order low.

\section{Acknowledgment}

This work was supported by a research grant from the Natural Sciences and Engineering Research Council of Canada.

\section{References}

${ }^{1}$ Balas, G. J., and Doyle, J. C., "Robustness and Performance Trade-offs in Control Design for Flexible Structures," IEEE Transactions on Control Systems Technology, Vol. 2, No. 4, 1994, pp. 352361 .

${ }^{2}$ Smith, R. S., Chu, C. C., and Fanson, J. L., "The Design of H-infinity Controllers for an Experimental Non-collocated Flexible Structure Problem," IEEE Transactions on Control Systems Technology, Vol. 2, No. 2, 1994, pp. 101-109.

${ }^{3}$ Madelaine, B., and Alazard, D., "Flexible Structure Model Construction for Control System Design," AIAA Paper 98-4316, Aug. 1998.

${ }^{4}$ Baldelli, D. H., and Sanchez Pena, R. S., "Uncertainty Modeling in Aerospace Flexible Structures," Journal of Guidance, Control and Dynamics, Vol. 22, No. 5, 1999, pp. 611-614.

${ }^{5}$ Boulet, B., Francis, B. A., Hughes, P. C., and Hong, T., "Uncertainty Modeling and Experiments in $\mathrm{H}$-infinity Control of Large Flexible Space Structures," IEEE Transactions on Control Systems Technology, Vol. 5, No. 5, 1997, pp. 504-519.

${ }^{6}$ McFarlane, D. C., and Glover, K., "Robust Control Design Using Normalized Coprime Factor Plant Descriptions," Lecture Notes in Control and Information Sciences, Springer-Verlag, London, 1990.

${ }^{7}$ Balas, G. J., Doyle, J. C., Glover, K., Packard, A., and Smith, R. S., “ $\mu$ Analysis and Synthesis Toolbox: User's Guide," MathWorks, Natick, MA, 1991.

${ }^{8}$ Yang, C. D., Chang, C. Y., and Sun, Y. P., "Mixed $\mu$-Synthesis via $H_{2}-$ Based Loop-Shaping Iteration," Journal of Guidance, Control, and Dynamics, Vol. 21, No. 1, 1997, pp. 182-184.

${ }^{9}$ Gawronski, K., "Balanced Control for Flexible Structures," Lecture Notes in Control and Information Sciences, Springer-Verlag, New York, 1995, Chap. 2.

${ }^{10}$ Kollar, I., "Frequency Domain Identification Toolbox," MathWorks, Natick, MA, 1997. 